\title{
PRODUCTION AND CHARACTERIZATION OF MONOCLONAL ANTIBODIES AGAINST THE NUCLEOCAPSID PROTEIN OF SARS-COV
}

\author{
Ying Fang, Andrew Pekosz, Lia Haynes, Eric A. Nelson, \\ and Raymond R. R. Rowland*
}

\section{INTRODUCTION}

To elucidate the basic mechanism of nucleocapsid $(\mathrm{N})$ protein function in the pathogenesis of the disease and in aid of developing diagnostic tests for SARS-CoV, we produced a panel of monoclonal antibodies (MAbs) against SARS-CoV N protein. We further tested their application to the detection of SARS-CoV infection using the methods of Western blotting, immunofluorescent staining (IFA), and ELISA.

\section{MATERIALS AND METHODS}

DNA encoding the $\mathrm{N}$ protein was amplified from cDNA prepared from the Urbani isolate (CDC) and cloned into the pBAD-TOPO protein expression vector (Invitrogen). The $\mathrm{N}$ protein was expressed in E. coli as a 6-His tagged fusion protein and purified using Ni-NTA columns (Qiagen). BALB/c mice were immunized with purified recombinant $\mathrm{N}$ protein at 2 -week intervals for 8 weeks. Splenocytes from immunized mice were fused with NS-1 myeloma cells and cultured on 24-well plates. Cell culture supernatants from wells containing hybridoma colonies were initially screened by ELISA using recombinant $\mathrm{N}$ protein. Hybridoma cells from positive wells were subcloned, expanded, and retested.

For Western blot analysis, Vero cells (ATCC) were mock-infected or infected with SARS-CoV (Urbani strain). The cell lysate was separated on a $15 \%$ polyacrylamide gel, transferred to PVDF membranes, and then incubated with an anti-N MAb. After extensive washing, the membranes were incubated with a HRP conjugated goat antimouse IgG (Jackson Laboratories). After 60 minutes, the blot was washed extensively

\footnotetext{
* Ying Fang, Eric A. Nelson, South Dakota State University, Brookings, South Dakota. Andrew Pekosz, Washington University School of Medicine, St. Louis, Missouri. Lia Haynes, Centers for Disease Control and Prevention, Atlanta, Georgia. Raymond R.R. Rowland, Kansas State University, Manhattan, Kansas.
} 
and visualized with ECL Plus Western blotting reagent (Amersham Biosciences) followed by exposure to autoradiographic film (Molecular Technologies).

For IFA, infected Vero cells were fixed with $2 \%$ paraformaldehyde at $24 \mathrm{~h}$ after infection and then permeabilized with PBS containing 3\% normal goat sera and $0.2 \%$ saponin (blocking buffer) for 15 minutes. MAbs were diluted 1:500 in blocking buffer and incubated with cells for $1 \mathrm{~h}$ at room temperature. After extensive wash, the cells were incubated with a goat anti-mouse IgG antibody conjugated to AlexaFluor 594 (Molecular Probes) or FITC (Jackson Laboratories) and the nuclear stain ToPro-3 (Molecular Probes). After incubation, the cells were washed and mounted onto microscope slides. Samples were analyzed on a Zeiss LSM 510 confocal microscope.

For ELISA, detergent extracted, gamma-irradiated SARS-CoV or recombinant SARS N protein were coated onto 96-well plates (Dynex) in bicarbonate buffer (pH 9.6) overnight at $4^{0} \mathrm{C}$. The plates were blocked with $2 \%$ BSA for $1 \mathrm{~h}$ at $37^{\circ} \mathrm{C}$. After washing, $100 \mu \mathrm{l}$ of serially diluted MAbs were added and incubated for $1 \mathrm{~h}$ at $37^{\circ} \mathrm{C}$, and then HRPgoat anti-mouse $\mathrm{IgG} / \mathrm{IgM}$ was added and incubated for 45 minutes. Plates were developed using ABTS, and OD values were determined using an ELISA plate reader.

\section{RESULTS AND DISCUSSION}

Initial hybridoma screening by ELISA using recombinant $\mathrm{N}$ protein yielded three candidate MAbs, SA 87-A1, SA 46-4, and SA 41-48. The isotypes of these MAbs were determined using an IsoStrip Kit (Serotech) and results showed that SA 87-A1 was an IgG1, SA 46-4 was an IgG2a, and SA 41-48 was an IgM.

We further tested the reactivity of these MAbs by IFA on Vero cells expressing recombinant EGFP-N protein. The SARS-CoV (Ubani strain) $\mathrm{N}$ gene was cloned into pEGFP-C2 vector (Clontech), and Vero cells were transfected with plasmid pEGFP-C2N. MAb SA 46-4 showed the highest sensitivity and specificity in recognizing the EGFP$\mathrm{N}$ protein in Vero cells, but the other two MAbs showed weak fluorescence and high background (data not show). To further confirm the specificity of MAb SA 46-4, it was tested for cross-reactivity with other coronaviruses and arteriviruses. It did not cross-react with porcine transmissible gastroenteritis virus (TGEV), mouse hepatitis virus (MHV), or porcine reproductive and respiratory syndrome virus (PRRSV).

We further explored the sensitivity and specificity of these MAbs for detection of SARS-CoV infection using Western blotting, IFA, and ELISA. Protein specificity of MAbs SA 87-A1 and SA 46-4 was first studied by Western blot analysis of Vero cell lysates infected with SARS-CoV. The results in Fig. 1 showed that MAbs SA87-A1 and SA46-4 reacted with $\mathrm{N}$ from infected cells and not with proteins from mock-infected cells. These MAbs reacted with a viral protein, which migrated as a doublet close to 50 $\mathrm{kDa}$, the predicted size for the $\mathrm{N}$ protein. The presence of $\mathrm{N}$ in two forms was also shown by Western blotting using in vitro expressed $\mathrm{N}$ protein (data not shown). The presence of $\mathrm{N}$ in two forms may reflect a post-translational modification. This result is consistent with the results reported by Leung et al. ${ }^{1}$ In their experiments, Western blots prepared using lysates from SARS-CoV infected cells probed with sera from patients showed several $\mathrm{N}$ protein-specific bands. They indicated that the $\mathrm{N}$ protein cleavage site is at the C-terminal, and this type of cleavage is specifically found in every preparation of the crude extracts. Post-translational cleavage of $\mathrm{N}$ would be a unique property of SARS$\mathrm{CoV}$, which is a subject of further study in our laboratory. 


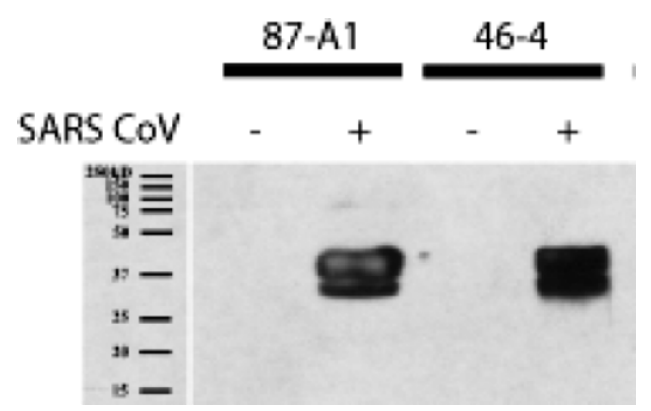

Figure 1. Detection of SARS-CoV N protein with Western blot. SARS-CoV viral lysates were electrophoresed with SDS-PAGE. After blotting on the PVDF membrane, proteins were detected by incubation with anti-N MAbs, followed by incubation with a horseradish peroxidase conjugated goat anti-mouse IgG. The blot was visualized with ECL Plus Western blotting reagent followed by exposure to autoradiographic film.

To further define MAb specificity, IFA was performed on Vero cells infected with SARS-CoV Urbani strain. The cells were fixed and incubated with MAbs SA87-A1 and SA46-4, and then double stained with AlexaFluor 594 (Molecular Probes) conjugated goat anti-mouse IgG and a nuclear stain ToPro-3 (Molecular Probes). As shown in Fig. 2, both MAbs SA46-4 and SA87-A1 specifically recognized the viral N protein, which is accumulated in the cytoplasm, in contrast with the ToPro-3 nuclear staining.

A commonly used method for the diagnosis of SARS-CoV infection is ELISA. To determine if these MAbs have any value for future development of serological tests, we tested MAbs SA46-4, SA87-A1, and SA41-48 in indirect ELISA using either in vitro expressed recombinant $\mathrm{N}$ protein or viral lysate as antigen. Antibodies were applied as twofold serial dilutions. Consistent with the other tests, MAb SA46-4 showed the highest sensitivity (Fig. 3).

Currently, although SARS-CoV appears to be under control, its future reemergence is still possible. Accurate and timely diagnosis of SARS infection is a critical step in preventing another global outbreak. Diagnostic reagents for SARS that are highly sensitive, specific, and convenient need to be developed. In this study, we produced a panel of anti-N MAbs, and MAb SA 46-4 consistently showed good sensitivity and specificity in all the tests conducted. Preliminary epitope mapping determined that MAb SA 46-4 recognizes an epitope located in the region between amino acids 50 to 200 .
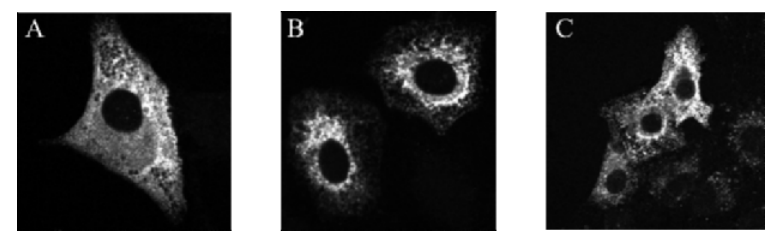

Figure 2. Confocal microscopy of SARS-CoV-infected Vero cells immunostained with anti-N MAbs SA 46-4 (A), SA 87-A1 (B), or SA 41-48 (C). Vero cells were parformaldehyde-fixed at $24 \mathrm{~h}$ postinfection. Fixed cells were incubated with anti-N MAbs and stained with AlexaFluor 594-labeled goat anti-mouse IgG (red). Cells were counterstained with the nuclear stain, ToPro-3 (data not shown). 

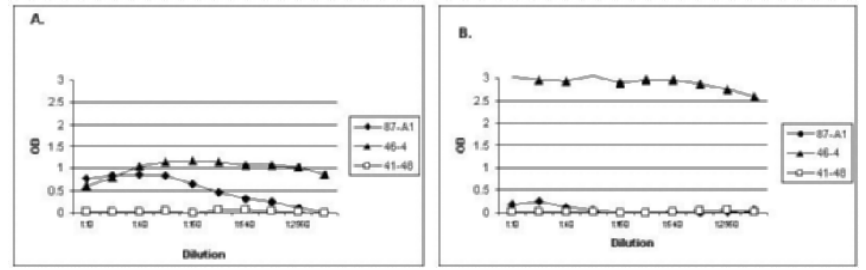

Figure 3. ELISA comparison of anti-N MAb reactivity to SARS-CoV (A) or to recombinant N protein (B). Detergent extracted, gamma-irradiated SARS-CoV or recombinant SARS N protein was coated onto 96-well Immunon-2 plates. Serially titrated anti-N MAbs were reacted for $1 \mathrm{~h}$ at room temperature and the bound MAbs were detected by peroxidase labeled goat anti-mouse $\mathrm{IgG} / \mathrm{IgM}$ followed by color development with ABTS.

Other anti-N MAbs have been reported. ${ }^{2,3}$ Some of them have been mapped to epitope sites on the $\mathrm{N}$ protein, and they recognize different epitopes than reported here. ${ }^{2}$ Previous studies showed that none of the antigenic epitopes reacted with all test sera from SARS patients. ${ }^{4}$ Therefore, a "MAb cocktail" combining currently available anti-N MAbs recognizing different antigenic epitopes could improve detection. Especially, because previous studies demonstrated that $\mathrm{N}$ protein detection exhibited a high positive rate between day 3 and day 5 after the onset of symptoms, these anti-N MAbs will be suitable reagents for early detection of SARS-CoV infection. ${ }^{5}$ Furthermore, the N protein has been determined to be the most antigenic structural protein, expressed in high abundance in infected cells, and $\mathrm{N}$ protein sequences are relatively conserved among different strains of SARS-CoV. ${ }^{1,4,6}$ These MAbs may have a substantial impact on the development of diagnostic tests for SARS.

\section{REFERENCES}

1. D. T. M. Leung, F. C. H. Tam, C. H. Ma, et al., Antibody response of patients with severe acute respiratory syndrome (SARS) targets the viral nucleocapsid, J. Infect. Dis. 190, 379-386 (2004).

2. Y. He, Y. Zhou, H. Wu, Z. Kou, S. Liu, and S. Jiang, Mapping of antigenic sites on the nucleocapsid protein of the severe acute respiratory syndrome coronavirus, J. Clin. Micro. 42, 5309-5314 (2004).

3. K. Ohnishi, M. Sakaguchi, T. Kaji, et al., Immunological detection of severe acute respiratory syndrome coronavirus by monoclonal antibodies, Jpn. J. Infect. Dis. 58, 88-94 (2005).

4. J. Wang, J. Wen, J. Li, et al., Assessment of immunoreactive synthetic peptides from the structural proteins of severe acute respiratory syndrome coronavirus, Clin. Chem. 49, 1989-1996 (2003).

5. B. Di, W. Hao, Y. Gao, et al., Monoclonal antibody-based antigen capture enzyme-linked immunosorbent assay reveals high sensitivity of the nucleocapsid protein in acute-phase sear of severe acute respiratory syndrome patients, Clin. Diag. Lab. Immun. 12, 135-140 (2005).

6. S. Li, L. Lin, H. Wang, et al., The epitope study on the SARS-CoV nucleocapsid protein, Genomics Proteomics Bioinformatics 1, 198-206 (2003). 